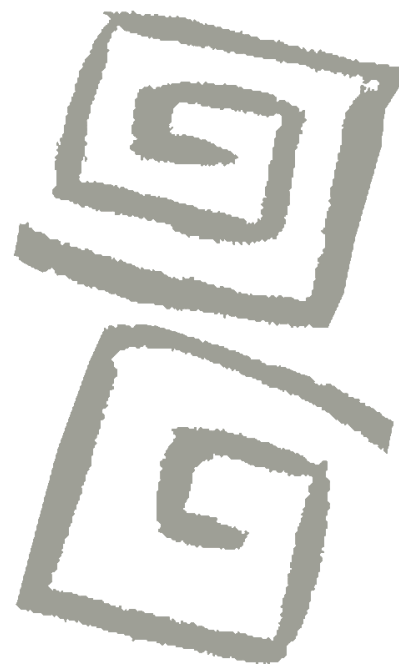

\title{
Guías bilingües: una estrategia para disminuir las barreras culturales en el acceso y la atención en salud de las comunidades wayuu de Maicao, Colombia
}

\author{
Bilingual guides: a strategy to decrease cultural barriers \\ to health care access in the Wayuu communities of \\ Maicao, Colombia
}

Sandra Yaneth Patiño-Londoño ${ }^{1}$, Javier Mignone ${ }^{2}$, Diana María CastroArroyave $^{3}$, Natalia Gómez Valencia ${ }^{4}$, Carlos Alberto Rojas Arbeláez ${ }^{5}$

\author{
${ }^{1}$ Antropóloga, Magíster \\ en Antropología. \\ Coinvestigadora, Universidad \\ de Antioquia, Colombia. \\ $\triangle$ (iD) \\ ${ }^{2}$ Psicólogo. Doctor \\ en Ciencias de Salud \\ Comunitaria. Profesor, \\ Department of Community \\ Health Sciences, Faculty of \\ Health Sciences, University \\ of Manitoba, Canadá. $\bowtie$ iD \\ ${ }^{3}$ Psicóloga, Magíster en \\ Salud Colectiva. Integrante \\ del Grupo de Estudios \\ en Pedagogía, Infancia \\ y Desarrollo Humano \\ (GEPIDH). Coinvestigadora \\ Universidad de Antioquia, \\ Colombia. $\triangle$ (iD) \\ ${ }^{4}$ Profesional en Sistemas \\ de Información en Salud. \\ Magíster en Epidemiología. \\ Coinvestigadora, Universidad \\ de Antioquia, Colombia. \\ $\square$ iD \\ ${ }^{5}$ Médico. Doctor en \\ Epidemiología. Investigado \\ principal, Profesor titular, \\ Universidad de Antioquia, \\ Colombia. \\ $\triangle$ (iD
}

RESUMEN Este artículo estudia la función del guía bilingüe como actor en la disminución de la brecha en el acceso y la atención en salud de las comunidades indígenas wayuu de Colombia. En el marco de un proyecto de VIH llevado a cabo entre los años 2012 y 2014, se realizaron 24 entrevistas a actores claves del área administrativa y de salud, incluyendo guías bilingües wayuu. A partir del análisis cualitativo se identificaron tres barreras culturales respecto al acceso a la atención en salud: a) idioma; b) cosmovisión wayuu sobre el cuerpo, la salud y la enfermedad; c) información sobre salud sexual y reproductiva y $\mathrm{VIH}$ culturalmente no adaptada. El estudio identifica al guía bilingüe como actor clave en la disminución de estas barreras y finaliza con una discusión sobre el rol de los guías, las tensiones inherentes a su labor, y la complejidad de su aporte como mediadores culturales.

PALABRAS CLAVES Salud Indígena; Atención a la Salud; VIH; Equidad en el Acceso; Asistencia Sanitaria Culturalmente Competente; Colombia.

\begin{abstract}
The article examines the use of bilingual guides to decrease cultural barriers to health care access in the Wayuu indigenous communities of Colombia. Within a larger project on HIV carried out between 2012 and 2014, 24 interviews were conducted with key actors in the administrative and health areas, including Wayuu bilingual guides. As a result of the qualitative analysis, the study identified three cultural barriers to health care access: a) language; b) the Wayuu worldview regarding the body, health, and illness; and c) information about sexual and reproductive health and HIV not adapted to the Wayuu culture. The study identifies the bilingual guides as key actors in reducing these barriers and concludes with a discussion of the role of the guides, the tensions inherent to their work, and the complexity of their contributions as cultural mediators.
\end{abstract}

KEY WORDS Indigenous Health; Health Care (Public Health); HIV; Equity in Access Culturally Competent Care; Colombia. 


\section{INTRODUCCIÓN}

El nivel de salud de los pueblos indígenas es comparativamente más bajo que el de la población general en las Américas, de acuerdo con diferentes indicadores como, por ejemplo, la mortalidad materno infantil ${ }^{(1)}$. Según la Organización Panamericana de la Salud, entre las causas de muerte más comunes en los pueblos indígenas se encuentran: malaria, tuberculosis, desnutrición, alcoholismo, drogadicción, VIH/sida y suicidio ${ }^{(2)}$. Estas deficientes condiciones de salud están asociadas a situaciones de pobreza y desigualdad social, así como a limitaciones en el acceso a la atención de la salud. Este artículo presenta una iniciativa de salud indígena wayuu de Colombia que, a través del uso de guías bilingües, intenta disminuir las barreras culturales en el acceso a la atención de la salud. Para ello, se analiza el rol de los guías, las tensiones inherentes a su labor, y la complejidad de su aporte como guías culturales.

El pueblo wayuu vive en La Guajira, Colombia, y en el oriente de Venezuela, en localidades cercanas al Caribe; es una de las 84 etnias indígenas de Colombia, de acuerdo con el Censo 2005. Es un pueblo amerindio de la familia lingüística arawak ${ }^{(3)}$. Se estima que la población wayuu en Colombia es de 402.279 personas, lo que representa el $19,98 \%$ de la población indígena de todo el país y el $45 \%$ del departamento de La Guajira. La mayoría vive en comunidades rurales a lo largo de toda la región. Las condiciones de vivienda varían poco entre las comunidades. El estilo principal de construcción son casas con marcos de madera (yotojoro) y paredes de barro. La mayoría de las viviendas no tienen agua corriente ni electricidad, aunque algunos hogares tienen generadores eléctricos. El acceso a agua potable y saneamiento es una problemática seria en las comunidades wayuu $^{(4)}$.

La tasa de analfabetismo en la población indígena en Colombia corresponde al 28,6\% en personas mayores de 15 años, frente al $7,4 \%$ de la población nacional ${ }^{(5)}$. En relación con la educación formal, solo el $46 \%$ de los indígenas logran terminar la escuela primaria completa ${ }^{(6)}$. En el caso específico de los wayuu, el $33 \%$ de los niños que comienzan la escuela finalizan el undécimo grado de educación media y aproximadamente el 18\% de los que viven en el área rural son analfabetos $\mathrm{o}$ analfabetos funcionales ${ }^{(7)}$.

En cuanto al estado de salud de los wayuu en Colombia, su perfil epidemiológico está ligado a factores socioeconómicos y culturales como la pobreza, la escasez de recursos, la geografía, la organización social y la actividad económica. Las enfermedades más frecuentes en este contexto son infecciones respiratorias, gastrointestinales y de transmisión sexual; cáncer uterino/cervical; y enfermedades asociadas a la malnutrición ${ }^{(8,9)}$. A esto se suman factores que afectan el acceso a la atención en salud, como las distancias geográficas que hay entre las comunidades y los centros de salud, y diferencias culturales que actúan como limitante en la interacción con el personal de salud.

Si bien los saberes de la medicina tradicional hacen su aporte a la salud de las comunidades gracias a su amplia variedad de terapias, producto de los conocimientos y prácticas basadas en experiencias y creencias indígenas ${ }^{(10,11)}$, el acceso a la medicina occidental (derecho explícitamente pautado a nivel constitucional en Colombia) presenta serias limitaciones ${ }^{(2)}$. Entre otros factores, para que el acceso a la atención en salud sea efectivo, el personal de salud -particularmente del primer nivel de atención, cercano a las comunidades- debe conocer y respetar las nociones de salud y enfermedad de los indígenas, así como los recursos que la cultura misma tiene para apoyar el proceso de atención. Las barreras culturales e idiomáticas entre el personal de salud y los usuarios indígenas dificultan esta situación.

El gobierno de Colombia aprobó en 1993 la Ley 100 que creó las empresas promotoras de salud (EPS). Esto resultó en la implementación de un sistema descentralizado de seguro de salud, con la expresa intención de lograr la cobertura universal hacia fines de la década de 1990. No obstante, la cobertura universal y equitativa tardó en lograrse y aún 
se discute si se ha alcanzado. Aunque las cifras son debatidas, las estadísticas oficiales indican que el $96 \%$ de la población tiene actualmente cobertura de seguro de salud( ${ }^{(4)}$.

Las EPS están financiadas por dos sistemas diferentes. Para segmentos de la población con suficientes recursos está el régimen contributivo. El régimen subsidiado cubre a las poblaciones de bajos recursos. Aquellos con empleo formal contribuyen con un $4 \%$ de su sueldo y el empleador contribuye con el 8,5\%. Los autónomos contribuyen con el 12,5\% del $40 \%$ de sus ingresos brutos. El régimen subsidiado está financiado por aportes que realizan los afiliados al sistema contributivo, así como por fondos estatales. La legislación estipula un Plan Obligatorio de Salud, administrado por las EPS, que cubre un paquete de intervenciones en salud. Estas incluyen servicios de promoción y prevención, así como de atención de baja, mediana y alta complejidad. Para la atención de sus afiliados, las EPS contratan servicios médicos y de promoción de la salud con instituciones prestadoras de salud (IPS) (centros de atención privados con o sin fines de lucro) ${ }^{(12)}$.

En 1997, organizaciones indígenas de diferentes regiones de Colombia crearon seis EPS indígenas (EPSI) del régimen subsidiado (sin fines de lucro). Una de ellas es Anas Wayuu, con sede en el municipio colombiano de Maicao, ubicado en el departamento de La Guajira. Esta EPSI fue creada por dos asociaciones que representan a 120 comunidades indígenas: la Asociación de Cabildos y/o Autoridades Tradicionales de la Guajira, y la Asociación Sumuywajat. Actualmente, Anas Wayuu cuenta con 129.098 afiliados, de los cuales el $80 \%$ es indígena, y el $20 \%$ no indígena. Esta EPSI es responsable de la cobertura de servicios de salud primarios, secundarios y terciarios, así como de programas de prevención y promoción. La mayoría de los empleados son de la etnia wayuu y hablan ambos idiomas wayuunaiki (lengua de los wayuu) y castellano. Anas Wayuu ofrece servicios de guías bilingües para las familias wayuu.

Con el establecimiento de las EPSI se esperaba reducir barreras en la atención en salud y apalancar iniciativas de salud intercultural que incluyeran la cosmovisión indígena en el proceso salud-enfermedadatención ${ }^{(7)}$. Un elemento central en este proceso es la figura del guía bilingüe o facilitador intercultural, como se lo conoce en otras regiones. Aunque es una figura común en la mayoría de los modelos de atención intercultural en salud, las descripciones detaIladas sobre su perfil son escasas. En general, se contemplan como requisitos fundamentales ser bilingüe y ser líder comunitario(13). Los guías bilingües son indígenas con competencias comunicativas sobresalientes en la escucha, el habla, la lectura y la escritura del castellano, y con dominio de su lengua nativa, aunque no necesariamente tengan competencias en su lectura y escritura, ya que no todas las lenguas indígenas cuentan con una gramática establecida. Usualmente, han tenido una educación occidental y han logrado profesionalizarse como auxiliares de enfermería, medicina, trabajo social, etc. Otros, por el contacto permanente con los no indígenas y debido a su rol dentro de la comunidad -por ejemplo, autoridades tradicionales- han logrado adquirir buenas competencias comunicativas. Más allá de cómo adquirieron la segunda lengua, todos comparten una característica fundamental y es la capacidad de comunicarse con el personal administrativo y de salud, debido a que su castellano es más especializado. Esto los hace actores claves dentro y fuera de los centros de atención en salud ${ }^{(14)}$.

Los guías bilingües vinculados a Anas Wayuu trabajan conjuntamente con el personal médico y administrativo. Dentro de la institución, brindan apoyo a las áreas de atención al usuario, asignación de citas, laboratorio, urgencias, consulta interna y externa e incluso apoyan la labor de los porteros, en el caso de que no sean wayuu. Asimismo, acompañan el triaje de los pacientes indígenas, para lograr un acceso oportuno. Los guías no solo prestan sus servicios en las cabeceras municipales, sino también en las zonas rurales, en el acompañamiento y sensibilización de las autoridades tradicionales, líderes y comunidad en general. Se encargan 
de apoyar al personal de salud con las charlas de promoción y prevención. Acompañan al paciente que está temporalmente en las casas de paso (albergues que permiten la estadía de los pacientes indígenas con el fin de facilitar el acceso a los diferentes niveles y la continuidad de la atención en salud de forma oportuna y adecuada culturalmente) brindándole asistencia en la interacción con el personal médico. Apoyan la identificación de la población beneficiaria potencial y acompañan a los pacientes wayuu que requieren atención especializada fuera de La Guajira, para superar la barrera idiomática y cultural. Dentro del espacio médico, los guías son los encargados de orientar al usuario wayuu que no habla castellano o que simplemente opta por hablar en su lengua con el personal de salud. Apoyan la gestión de documentos del paciente, los cuales están escritos en castellano. También ingresan, en calidad de intérpretes, al escenario de la consulta médica, y se encargan de explicar cada medicamento indicado, tiempo de consumo y forma de uso.

El presente artículo analiza información recogida por el proyecto Prevención, diagnóstico y tratamiento del VIH/sida con COmunidades wayuu de Maicao-La Guajira (2012-2014), para estudiar en profundidad el rol del guía bilingüe en el trabajo con población indígena. Este proyecto se llevó a cabo con 55 comunidades wayuu ubicadas en el área rural del municipio de Maicao, en La Guajira, y con instituciones de salud del área urbana de este municipio.

La figura de guías bilingües no es nueva, se ha recurrido a ellos desde hace muchos años en diversos países, en una variedad de campos, como el educativo ${ }^{(15)}$ y la salud mental ${ }^{(16,17)}$ en la atención de la salud de inmigrantes $^{(18)}$ e indígenas ${ }^{(19)}$. Estudios sobre guías bilingües en la atención de la salud han confirmado su impacto positivo cuando los guías están bien capacitados ${ }^{(20)}$.

Estudios previos han identificado que los guías bilingües son mucho más que meros traductores de palabras. Son más bien intérpretes de cultura ${ }^{(18)}$, porque tienen la capacidad de observar lo que es inobservable para quienes son de otra cultura ${ }^{(21)}$. Su tarea es la de comunicar sentidos sutiles, expresiones idiomáticas, significados implícitos, tonos de voz, expresiones faciales, además de las verbales ${ }^{(22)}$. Más aún, la de interpretar sistemas de conocimientos y creencias mayormente discordantes ${ }^{(19)}$ debido a su capacidad de transitar entre dos mundos; en este caso, el de las creencias wayuu y el del médico-normativo-occidental. El artículo estudia la función del guía bilingüe como actor en la disminución de la brecha en el acceso y la atención en salud de las comunidades indígenas. A la vez, como facilitador en la reducción de barreras culturales para abordar temas como la salud sexual y reproductiva, y el $\mathrm{VIH} /$ sida en el contexto wayuu.

\section{METODOLOGÍA}

El proyecto Prevención, diagnóstico y tratamiento del $\mathrm{VIH} /$ sida en comunidades wayuu de Maicao-La Guajira (2012-2014) combinó estrategias de investigación cuantitativa y cualitativa para responder a dos grandes retos: el análisis epidemiológico del $\mathrm{VIH} /$ sida y el diseño e implementación de un plan de intervención comunitaria para la prevención del VIH. El estudio contó con el aval del Comité de Ética de la Facultad Nacional de Salud Pública de la Universidad de Antioquia y de un comité indígena con representantes de Anas Wayuu y otras entidades indígenas aliadas al proyecto. El estudio fue financiado por Colciencias (entidad del gobierno nacional de Colombia que financia la investigación científica) y fue formulado por invitación de la EPSI Anas Wayuu y la IPS asociadas (Asocabildos, Sumuywajat y Mediser) junto con investigadores de la Universidad de Antioquia, Medellín, Colombia y la University of Manitoba, Winnipeg, Canadá. Las entidades indígenas también brindaron apoyo económico y logístico en calidad de co-ejecutores. El equipo trabajó de la mano de las autoridades indígenas y las 55 comunidades, por tratarse de una investigación basada en la comunidad. 
Se formularon y se siguieron protocolos de investigación para asegurar el respeto a las voces de las distintas entidades. Los resultados del estudio fueron compartidos, aunque las interpretaciones formuladas en este texto son responsabilidad de los autores.

Este artículo busca reflexionar en torno a las barreras culturales que pueden presentarse en el encuentro entre pacientes o usuarios indígenas y el personal de salud, cómo estas pueden ampliar la brecha de acceso a los servicios de salud, especialmente a los relacionados con la salud sexual y reproductiva y, finalmente, de qué manera el rol del guía bilingüe en este escenario podría llegar a disminuir esa brecha. Usualmente, este tipo de barreras se dan cuando el vínculo entre la persona y el sistema de salud se ve obstaculizado por las creencias, idioma, conductas, símbolos o tradiciones ${ }^{(23)}$, afectando consecuentemente el acceso a los servicios de salud.

Con previo consentimiento informado, en el año 2012 se realizaron entrevistas semiestructuradas al personal de salud de diferentes instituciones que forman parte de la red de prestadores de la EPSI Anas Wayuu. También se utilizaron guías de observación durante las visitas y reuniones con el personal de salud y las comunidades.

Se realizó un muestreo por conveniencia y se llevó hasta el nivel de saturación de la información ${ }^{(24)}$, teniendo en cuenta la disposición de las personas para participar y la calidad de la información suministrada por ellas. Se visitaron nueve instituciones de salud; de las cuales cinco eran indígenas. Allí se entrevistó a un total de 24 participantes, actores claves del área administrativa y de salud, con edades entre 23 y 48 años; del total 14 eran mujeres y ocho eran guías bilingües wayuu.

Posteriormente, se analizaron las 24 entrevistas con asistencia del software Atlas ti versión 6.2 , bajo la perspectiva de categorías emergentes. Una vez transcritas las entrevistas se volvió sobre los datos para explorar el sentido general, y para organizarlos en primer lugar con relación al actor clave $y$, posteriormente, al lugar de este dentro del contexto. Se agrupó al personal de salud no indígena, en otro grupo al personal indígena, luego al personal administrativo no indígena y, por último, a los guías. Se analizaron las relaciones al interior de cada grupo y entre las agrupaciones. Esto permitió identificar encuentros y desencuentros entre los diferentes actores. Se registraron las categorías emergentes y su relación con el planteamiento del problema. Posteriormente, se describió e interpretó el significado de las categorías y se integraron aquellas que tenían similitudes.

\section{RESULTADOS}

Durante el proceso de análisis emergieron categorías que dieron lugar a la organización y comprensión de los datos. Esto posibilitó identificar tres barreras culturales respecto al acceso a la atención en salud tales como: a) idioma, b) cosmovisión wayuu sobre el cuerpo, la salud y la enfermedad, y c) información sobre salud sexual y reproductiva y VIH culturalmente no adaptada. A continuación presentamos dichas barreras enfatizando el rol del guía como actor en la disminución de estas.

\section{El idioma como barrera cultural y el guía bilingüe como mediador en la comunicación}

Según explica un entrevistado, el idioma es una barrera bidireccional entre el paciente o usuario indígena y el médico o personal administrativo:

\footnotetext{
Yo pienso que una de las mayores barreras es cuando ellos no hablan nada de castellano, esa es una de las barreras más grandes, porque cuando existe el acompañamiento y hay la interpretación, son pocas las dificultades. (Mujer, no-indígena, médica. Entrevista, septiembre 2012)
} 
Pero no se trata solo de la falta de competencias comunicativas en los indígenas, sino también por parte del personal de salud occidental que no cuenta con competencias suficientes en wayuunaiki, ya que la gran mayoría de ellos llega de otras zonas del país sin contacto previo con la cultura wayuu. Igualmente, el personal occidental originario de La Guajira, aunque interactúa en forma regular con personas wayuu, no conoce el idioma. No obstante, algunos de los que llevan varios años trabajando en la zona han aprendido algunas frases que, aunque ayudan, pueden no ser suficientes para llevar a cabo una consulta médica:

Ya con el tiempo uno aprende a preguntarle en wayuu, y es que generalmente ellos, pues te dicen "ais tekii", así "ais tekii" mira te están diciendo me duele la cabeza "ais tekii", "ais tanut" me duele el cuello, "ais toú" me duele el ojo, entonces hacen así y al médico ya le va quedando eso, y al próximo "ais tekii", ya él sabe qué le duele. Hay que aprendernos a entender, eso es clave, pero sí se puede, ya pues él entiende, "ais supula tashituin", le duele al orinar, entonces ya eso se vuelve, pues repetitivo y el médico va asociando. (Hombre, noindígena, administrador de empresas. Entrevista, octubre 2012)

Por otro lado, a pesar del contacto permanente con los alijunas (no indígenas), un buen segmento de los usuarios wayuu no tienen competencias en castellano o, las tienen, pero con limitaciones. Por ejemplo, hablan en castellano pero no lo leen. Los jóvenes y niños mayormente hablan y escriben en castellano por el carácter bilingüe de la educación, pero en los grupos etarios superiores, las brechas son significativas. Este escenario se complejiza cuando el wayuu que entiende castellano no desea hablarlo ya sea por vergüenza, miedo o desinterés. Finalmente, hay pacientes wayuu que se comunican en castellano, pero no cuentan con las competencias para comprender el lenguaje especializado del médico:

\begin{abstract}
Aquí llega el wayuu con su acudiente, o sea, con un acompañante en caso que, de pronto, no hable muy bien castellano y le dé miedo, porque de eso tiene mucho el wayuu, de que le da miedo. (Mujer, wayuu, trabajadora social, guía bilingüe. Entrevista, septiembre 2012)
\end{abstract}

La barrera lingüística entre el indígena y el personal de salud se vivencia en forma plena en el espacio de la relación médicopaciente. El paciente intenta expresar verbalmente el dolor o el malestar que carga su cuerpo, transformando la palabra en el inicio de la sanación. Sin embargo, este escenario ideal se ve truncado cuando la comunicación es limitada. El wayuu que no habla castellano, o solo un poco, se ve en la necesidad de tener un intermediario que logre interpretar aquello que dice el médico $y$, a la vez, le comunique a este los males que le aquejan. Pero esta necesidad también es sentida por el personal de salud:
No tengo la expresión para intercomu- nicar con ellos y se pierden muchas cosas y me toca llamar a la guía para que me haga la traducción. (Hombre, no-indígena, médico. Entrevista, octubre 2012)
El idioma es una barrera, o sea, pues no todo el mundo tiene la posibilidad de en- tender algo del idioma. (Mujer, wayuu, médica. Entrevista, octubre 2012)

Algunos médicos occidentales han logrado familiarizarse con algunas expresiones del wayuunaiki, por ejemplo: "kasaichi pünülia"? ¿cómo se Ilama?, "Püshakata" ¡bájate!, "Püjülerra" jacuéstate! Sin embargo, ese saber es insuficiente y requieren del acompañamiento del guía bilingüe. La barrera idiomática y la ausencia del guía en la consulta generan que muchos pacientes no entiendan adecuadamente qué tipo de padecimiento tienen, cómo y cuándo deben tomar sus medicamentos, o en qué consiste el examen de laboratorio, entre otros procedimientos. Una médica wayuu comentaba: 
Las wayuu no sabían nada de castellano, había una wayuu que venía juiciosita a buscar su tabletica de anticonceptivos orales, su cajita, entonces cuando era inyectable ella vino y me dijo mira estoy sangrando, esto no sé qué, entonces uno le explicaba en wayuunaiki y yo decía: jay Dios mío!, pero si este [médico] no le explicó, claro, después... no, que ella no se lo tomó, la verdad es que él no le explicó, él no le explicó. (Mujer, wayuu, médica. Entrevista, octubre 2012)

Muchos de los wayuu que recurren a la medicina occidental saben algo de casteIlano. Los que no, mayormente llegan a los centros de atención acompañados con familiares bilingües. Sin embargo, el lenguaje médico suele ser tan especializado que el castellano que dominan (tanto el paciente como su familia) no basta y se ven en la necesidad del guía bilingüe, quien cumple la función de intérprete especializado. Respecto a la relación médico-paciente, los guías bilingües señalan que los usuarios les agradecen su intervención, ya que habitualmente no logran entender al médico. De hecho, incluso los que entienden y hablan bien el castellano a veces solicitan su presencia. El wayuu bilingüe promedio no cuenta con un castellano especializado en lo que al área de la salud se refiere, así que el apoyo del guía es un plus de la consulta médica:

En algunos casos [piden] que uno los acompañe, porque va a ser muy difícil, él no le va a entender a ella, en la mayoría de los casos ellos dicen: no, acompáñame, yo necesito que tú me acompañes, hasta las mujeres embarazadas me han pedido que pase con ellas. (Hombre, Wayuu, trabajador social, guía bilingüe. Entrevista, octubre 2012)

\section{Cosmovisión wayuu sobre el cuerpo, la salud y la enfermedad, y el guía bilingüe como puente entre la cultura ancestral y el quehacer médico}

La cosmovisión wayuu sobre el proceso salud-enfermedad-atención o la construcción cultural del cuerpo son desconocidos para la mayoría del personal de salud. Para los wayuu, la salud tiene una relación directa con el equilibrio entre el cuerpo, el espíritu, las deidades, las relaciones con los otros y el territorio; una ruptura o desequilibrio de alguno de estos componentes genera las enfermedades. Estas se clasifican de acuerdo a su origen natural (ayuulee) o sobrenatural (wanülüü). Entre ellas, se destacan las producidas por el agua o el aire, que no generan angustia -como una gripe-, o por contacto con cosas sucias como osamentas y animales; las "angustiosas", causadas por un contacto con el mal; y "las de afuera", que son producto de la interacción con los alijunas (no indígenas), como es el caso del $\mathrm{VIH}$. El sistema médico tradicional wayuu cuenta con médicos tradicionales (piachis) con diferentes especialidades que realizan rituales y prácticas terapéuticas que buscan recobrar el equilibrio perdido y curar la enfermedad, apoyados en las plantas medicinales, los sueños y los espíritus protectores ${ }^{(3,11,25,26)}$.

Existen diferencias entre el paciente wayuu y el paciente no indígena. Uno de los rasgos más sobresalientes es la manifestación corporal del dolor. Entre los wayuu, especialmente las mujeres, está mal visto gritar y/o quejarse. El dolor se soporta en silencio y esto se enseña a las jovencitas desde el encierro (ritual de paso de niñas a mujeres). Por ejemplo, en la sala de urgencias puede encontrarse a una mujer no indígena gritando durante el trabajo de parto $y$, a su lado, en completo silencio y sin manifestaciones físicas de dolor, a una wayuu en la misma situación. Este rasgo facilita que el personal de salud pase por alto a la wayuu y atienda primero a la no indígena. Esto ha generado, en consecuencia, algunos partos de mujeres wayuu en la sala de espera. Este rasgo cultural, y muchos otros, son bien conocidos por 
los guías bilingües. De ahí la importancia de su rol junto al médico en la clasificación primaria de los pacientes, ayudando a asegurar el acceso oportuno a la atención en salud. En esta misma línea, las construcciones culturales del cuerpo y la sexualidad, así como el rol social de la mujer wayuu, marcan considerablemente la relación médico-paciente. Esas construcciones pueden generar barreras de acceso a la atención en salud.

Cuando la wayuu está con el médico, él le dice: "te tienes que bajar [ropa interior] para hacer el tacto", entonces ella como que es reacia a ese tacto, entonces yo le digo: "no te preocupes que él no te va a ver". Y yo le digo al médico: "doctor, haga el proceso, pero no la vea", entonces él lo que hace es utilizar una sabanita entre las piernas y ella se siente en más confianza de que no le van a ver sus partes y el médico lo que hace es introducir su mano y hacer su tacto y el mira a su otro lado y ella ya pues se queda tranquila. (Mujer, wayuu, trabajadora social, guía bilingüe. Entrevista, septiembre 2012)

De allí la relevancia del guía bilingüe en esa relación, pues su propio bagaje cultural e institucional le facilita el tránsito entre la cosmogonía ancestral y la institucionalidad occidental, favoreciendo la atención en salud culturalmente adaptada. Por esto, el guía, además de cumplir un rol destacado al facilitar el acercamiento a la institucionalidad médica, favorece el encuentro entre el wayuu y el personal administrativo en salud. Fuera del consultorio, el guía se encarga de orientar al usuario, facilitando los trámites, la adjudicación de citas, la entrega oportuna de medicamentos y su administración, y el acompañamiento en las casas de paso, entre otras funciones. Esta relación cercana con los usuarios favorece el surgimiento de relaciones estrechas con el guía; convirtiéndose en un agente socializador, un depositario de confianza para el indígena que llega con algún malestar. Es tal su importancia en el acceso a la atención en salud que algunos pacientes prefieren regresar otro día al centro de atención en salud para ser atendidos personalmente por ellos.

De este modo, queda en evidencia que tanto los conocimientos que tiene el guía bilingue sobre los wayuu como la relación de confianza que este establece con el médico o profesional de la salud son fundamentales para facilitar la comunicación entre pacientes indígenas y médicos pues, como puede verse en el testimonio anterior, el guía no solo es un traductor, sino también un facilitador del proceso de atención.

\section{Información sobre salud sexual y reproductiva y VIH culturalmente no adaptada y el guía bilingüe como facilitador de información culturalmente adecuada}

Hay brechas en el acceso a información sobre salud sexual y reproductiva. Por un lado, el personal de salud cuenta con la información, pero no siempre cuenta con el conocimiento cultural e idiomático. El personal bilingüe tiene la capacidad de comunicarse con sus comunidades, pero sus creencias pueden no facilitar la transferencia de esa información:

\begin{abstract}
Hay como una brechita, porque la persona que le entrega esa información, como los médicos que son los que manejan salud sexual y la enfermera, no hablan directamente wayuu. (Mujer, no-indígena, coordinadora asistencial. Entrevista, septiembre 2012)
\end{abstract}

El acercamiento de los guías bilingües a las comunidades, para abordar temáticas de salud sexual y reproductiva (y específicamente VIH), es relativamente nuevo. Antes, sus funciones tenían un tinte más operativo, incluso administrativo, de apoyo en jornadas de prevención o repartiendo folletos, pero no se abordaban abiertamente estas temáticas con sus comunidades. A raíz del proceso de formación llevado a cabo por el proyecto de $\mathrm{VIH}$, los guías recibieron herramientas 
pedagógicas y de investigación para tratar estas temáticas que son tabú dentro de su cultura. Los guías bilingües se enfrentaron con la disyuntiva entre la información que deben brindar a los usuarios como profesionales de la salud y sus propias creencias culturales y religiosas bastante arraigadas sobre la sexualidad. Manejar este conflicto fue dificultoso:

El uso del condón siempre es un poco difícil de abordar y más si tenemos con qué colocarlo, yo mismo me siento como que... miércoles [sic] ¿yo voy a agarrar ese aparato? [Modelo de pene sintético] No sé si es por la cultura, pero al principio cuando yo veía eso... miércoles, esa cosa y todo el mundo me ve así. (Hombre, wayuu, trabajador social, guía bilingüe. Entrevista, octubre 2012)

Le comenté [a la persona que vive con VIH] sobre la enfermedad, de los riesgos que había, qué es la infección en sí, o se lo toma lo más normal, o sea, no es una persona que se va a deprimir o está tirado ¡no! Es una persona muy diferente a muchas cosas que uno veía en televisión. (Hombre, wayuu, trabajador social, guía bilingüe. Entrevista, octubre 2012)

El acompañamiento permanente en las comunidades por parte del equipo del proyecto de VIH (médicos, epidemiólogos, psicóloga y antropóloga) con experiencia en el abordaje de grupos indígenas y la experiencia propia del campo permitieron que los guías bilingües pudieran abordar más abiertamente temáticas de las cuales, en la cultura wayuu, "no está bien visto" hablar en público. Los guías tuvieron un rol activo y fundamental en los encuentros educativos con las comunidades, lideraron conversaciones en su lengua sobre el uso del condón, generalidades sobre infecciones de transmisión sexual y profundizaron en la prevención del VIH. Se pudo contrastar la diferencia en la actitud de los wayuu frente a la recepción de estas temáticas; usualmente, cuando el personal de salud occidental llega a las comunidades para abordar la salud sexual y reproductiva, los hombres y mujeres wayuu se ríen y hacen comentarios jocosos en wayuunaiki; sin embargo, cuando esta información fue brindada por un guía, en su propia lengua, los asistentes asumieron un rol más activo, participaron y aportaron desde sus propias experiencias.

Esas charlas estuvieron enriquecidas con las experiencias previas en trabajo comunitario de los guías y la tradición oral de su cultura. Por ejemplo, se comenzó con el uso de personajes míticos como Pulowi (una sirena de laguna) y Kashí (la luna), así como otros relacionados con la cotidianidad del wayuu, como el pastor de chivos o la tejedora de mochilas, para dar cuerpo a escenarios donde las prácticas de autocuidado y prevención eran el centro de la narración. Una función emergente de los guías interculturales es la del acompañamiento a las personas que viven con VIH. El proceso de formación les brindó las herramientas para tener un rol más activo con esta población. El trabajo estaba enfocado en garantizar la adherencia al tratamiento de las personas que viven con $\mathrm{VIH}$ -la asistencia a chequeos médicos, el manejo y consumo de los antirretrovirales, consejería psicológica, entre otras actividades- y en la búsqueda activa de nuevos casos por medio del uso de pruebas rápidas.

Ya la parte institucional, lo que es trabajo
social y psicología, ellos ya le hablaron
del tema [a la persona que vive con
VIH] pero yo quería, o sea yo sentía, yo
tenía la sensación de que yo tenía que
hablar, pero en mi lengua, en mi cultura,
mirar a ver cómo él se estaba sintiendo.
(Hombre, wayuu, trabajador social, guía
bilingüe. Entrevista, octubre 2012)

En los diferentes testimonios quedó claro que el guía bilingüe se constituye en un agente activo dentro del sistema de salud, que no está solamente para ayudar a su hermano indígena sino también al profesional de salud que reconoce sus falencias y limitaciones frente a una cultura que apenas conoce. 


\section{DISCUSIÓN}

El rol del guía bilingüe está enmarcado en una temática más amplia: la salud intercultural. Esta problemática viene siendo estudiada desde hace años en las Américas ${ }^{(27)}$. Entre los determinantes sociales de la salud y las diversas barreras al acceso de la atención en salud de los pueblos indígenas, el aspecto sociocultural es uno de los predominantes ${ }^{(28)}$. Investigaciones más recientes plantean que las iniciativas más plenas en salud intercultural son aquellas que son diseñadas y lideradas por organizaciones indígenas ${ }^{(29)}$. Si la interculturalidad en salud es entendida como prácticas en atención de la salud que hacen de puente entre la medicina tradicional indígena y la medicina occidental ${ }^{(29)}$, el guía bilingüe es un actor clave en este proceso. En el caso específico de los wayuu, estudios en la región concuerdan en señalar que el idioma no permite una comunicación eficaz y fluida en el ámbito de la educación en salud; además, la oferta de dos tipos de medicina desencadena indecisiones, ansiedad y deserción, que se traducen en procesos terapéuticos y preventivos inconclusos ${ }^{(11)}$. Estas y otras barreras del orden cultural son inherentes a muchos grupos indígenas de la región y se suman a los factores que amplían la brecha de acceso y atención en salud. Este artículo identificó la función del guía bilingüe como actor clave en la disminución de este tipo de brechas. A la vez, como facilitador en la reducción de otras barreras culturales para abordar temas de salud sexual y reproductiva, y del $\mathrm{VIH} /$ sida, en el contexto wayuu.

El estudio sugiere que la propia cultura proporciona recursos que pueden facilitar escenarios de salud y bienestar, siendo los guías bilingües un ejemplo de estos. Los guías son actores que juegan un papel esencial en la disminución de estas barreras. Lo hacen desde su función de puente entre las comunidades y las instituciones a partir del conocimiento de su cultura como de lo normativo e institucional, lo que les permite desenvolverse en los dos mundos. Aunque ser bilingües no asegura tener un mejor conocimiento sobre salud y enfermedad que los indígenas no bilingües, sí proporciona ventajas al momento de comprender mensajes provenientes de los agentes de salud ${ }^{(30)}$.

Como plantea la Organización Panamericana de la Salud: "cada pueblo indígena tiene sus creencias y prácticas únicas en lo referente a la salud, así como sus propios recursos comunitarios para la promoción de la salud, la prevención de enfermedades o la cura de los males comunes"(2 p.33). Estas creencias y prácticas suelen ser pasadas por alto por el personal médico. Un rol del guía bilingüe es interpretar palabras o gestos que están fuera de la comprensión no indígena y son claves para entender el padecimiento. En particular, al interior del consultorio se requiere una comunicación profunda en la que confluyan los lenguajes verbal y corporal, posibilitándole al médico llegar al diagnóstico.

A pesar de que las EPSI intentan construir un modelo intercultural de salud, que sea culturalmente más cercano para el usuario indígena, están dentro del marco de la medicina occidental y las instituciones prestadoras de los servicios no siempre son indígenas. Por lo tanto, están sujetas a una serie de códigos, normas y condicionantes propios de la institucionalidad no indígena y ajenas al dominio de los wayuu. Una de las fortalezas de una EPS indígena frente a una no indígena es la posibilidad de atención en salud en su lengua materna. Esto implica un conocimiento de sus costumbres y prácticas culturales, por ende, una comprensión de lo que significa "estar enfermo" y las consecuencias de ello dentro de su cosmogonía. Sin embargo, las guías y protocolos de atención en salud en Colombia no están adaptadas para los diferentes entornos culturales. El reto institucional es adaptarlas a su contexto y en este caso el guía bilingüe puede ser de gran ayuda.

El ideal de las EPSI es contar con personal médico indígena cualificado, para que sea más asertiva la comunicación y, por ende, la atención de los pacientes indígenas. Sin embargo, en La Guajira son escasos los médicos y las enfermeras wayuu. Solo en el ámbito de auxiliares de enfermería se encuentra 
un mayor número de ellos. No obstante, es aún insuficiente para la amplia demanda de usuarios indígenas. De ahí la importancia acentuada de contar con guías bilingües. Por otra parte, los profesionales indígenas se forman en un sistema occidental que considera poco o nada la realidad de grupos étnicos al momento de educar o poner en práctica la profesión.

El rol del guía bilingüe se ha operacionalizado con el paso del tiempo. Anas Wayuu ha encontrado más formas de incorporarlos dentro de la dinámica de atención, promoción y prevención de la salud. Ahora los guías bilingües salen del espacio institucional y viajan a las rancherías o comunidades con el programa extramural para realizar esas acciones en su propia lengua. El guía bilingüe es una fortaleza en el modelo de atención de las EPSI, ya que disminuye las barreras entre el paciente y la prestación de servicios de salud. Con ellos se articula la prestación inmediata en la atención institucionalizada. Sin embargo, se observa que esta figura no fue propuesta con las características y funciones que ahora pueden ofrecer, sino que el proceso y el lugar que han ido tomando en la práctica ha llevado a una valoración importante de estos actores por parte de las instituciones de salud.

A lo largo del artículo se ha resaltado el papel de los guías bilingües en la promoción de la salud. Sin embargo, en el campo de la salud sexual y reproductiva se requiere de un acompañamiento para su formación y cualificación. Esto obedece a que "la sexualidad es una construcción producto de sistemas sociales y culturales que moldean tanto la experiencia sexual, como las formas en que la entendemos e interpretamos" ${ }^{\prime \prime 31}$ p.86). Esto quiere decir que "cada sociedad genera costumbres y normas, prácticas y creencias, que regulan la expresión sexual, sobre la temporalidad o cuándo tener relaciones sexuales, con quién tenerlas, cuántas veces, de qué manera, con qué objetivo $y$, sobre todo, qué tipo de relaciones"(31 p.82). El guía bilingüe no escapa a las construcciones socioculturales de aquello que está mal o bien visto en su cultura, y ese "deber ser" influye directamente en la información sobre sexualidad que brinda a su comunidad y el modo en que lo hace, como en el caso del $\mathrm{VIH}$.

El proceso de formación de los guías bilingües en nuestro proyecto tuvo en cuenta este aspecto, ya que buscábamos que la información sobre infecciones de transmisión sexual y VIH llegara en wayuunaiki, tanto a hombres como a mujeres, de diferentes edades. Para garantizar eso necesitábamos que los guías estuvieran sensibilizados y concientizados de su rol en la transmisión de nuevo conocimiento sin ponerlos entre la espada y la pared respecto a sus creencias y costumbres. Por ello, un proceso de formación en temas de sexualidad y salud reproductiva con guías bilingües exige hacer conscientes emociones en torno a la sexualidad y asumir que lo que se requiere es un diálogo en el que también los investigadores se forman con los guías bilingües. Por ejemplo, hablar del tema con los "antiguos", como se refieren a los más ancianos, sin sentir que es una falta de respeto, fue un punto clave para investigadores y guías. También, saber cómo abordar el tema del uso del condón de forma individual y grupal, identificar qué temáticas se hablan entre hombres y cuáles entre mujeres. A su vez, aprender cómo presentar el tema de la homosexualidad adecuadamente, así como la convivencia con las personas que viven con VIH. En última instancia, a partir de esa de-construcción de tabúes, así como de sesgos personales, dar paso a la transferencia de nuevos conocimientos que apuntan a tener comunidades más saludables.

La importancia y la trascendencia del rol del guía bilingüe wayuu en la relación usuario indígena y médico muestra ciertas características del guía bilingüe que reproduce el rol del palabrero o pütchipü'ü de la cultura wayuu. Posiblemente, esos rasgos familiares han hecho que algunos indígenas que visitan los centros de atención en salud se valgan de ellos para "Ilevar la palabra" ante la institucionalidad, representada por el médico o el personal administrativo. Según Guerra ${ }^{(32)}$, dentro de la cultura wayuu, el palabrero es una persona que "ha adquirido prestigio por su don de la palabra", conoce muy bien la ley 
wayuu y cuenta con habilidades destacadas en la resolución pacífica de conflictos o desencuentros. "Es un personaje generalmente respetado y con la legitimidad para servir de intermediario y en casos más complejos de mediador" $^{\prime \prime 32)}$.

Ese lugar privilegiado en la intermediación lo encontramos también en la figura del guía bilingüe. Por ejemplo, los más experimentados de ellos son buscados por los usuarios para que hablen, en su nombre, ante el médico o el personal administrativo cuando surge algún problema o inconveniente en la prestación de un servicio de salud. Las acciones del guía reproducen la negociación wayuu, pero al interior de la institución de salud. Domina el mundo de las normas y el deber ser, en este caso la institucionalidad, conoce la cultura, las jerarquías, tiene una posición de poder y, ante los ojos de los pacientes, es su portavoz.

Un rasgo sobresaliente de los usuarios wayuu al interior de las instituciones de salud, en comparación con los pacientes no indígenas, es que no se quejan. No es común encontrar a un wayuu en la oficina de atención al usuario hablando sobre sus inconformidades frente al sistema de atención. Este rasgo tiene un cimiento cultural, puesto que dentro de los códigos de la negociación wayuu hay una figura que se encarga de "Ilevar la palabra", de transmitirle al otro las discrepancias o aquellas cosas que no están bien. Por ello, el guía se transforma en ese portavoz especializado que, desde una relación más equitativa de poder con el médico, puede expresar el malestar, la inconformidad o el descontento.

\section{AGRADECIMIENTOS}

Agradecemos a la Facultad Nacional de Salud Pública de la Universidad de Antioquia y a Colciencias por financiar el proyecto "Prevención, diagnóstico y tratamiento del $\mathrm{VIH} /$ sida en comunidades wayuu de Maicao, La Guajira (2012-2014), (código 111554531493); y a la University of Manitoba de Canadá. Igualmente, a la EPSI Anas Wayuu, a las IPS Asocabildos, Sumuywajat y Mediser y a la estrategia de sostenibilidad CODI de la Universidad de Antioquia. A las 55 comunidades wayuu de Maicao, La Guajira, Colombia y a sus autoridades tradicionales por haber participado y acogido el proyecto. Gracias a la Secretaría de Salud, al Hospital San José de Maicao, a las instituciones educativas, a los profesores, estudiantes y asesores; y a los líderes indígenas y contratistas que contribuyeron de diferentes maneras al proyecto.

\section{REFERENCIAS BIBLIOGRÁFICAS}

1. Montenegro RA, Stephens C. Indigenous health in Latin America and the Caribbean. Lancet. 2006;36:1859-1869.
2. Organización Panamericana de la Salud. Una visión de salud intercultural para los pueblos indígenas de las Américas [Internet]. Washington DC: OPS; 2008 [citado 10 ago 2015]. Disponible en: http://goo.gl/b3F9tb.

3. Díaz R. Métodos de profilaxis wayuu asociados a la tuberculosis: Significados y funciones. Fermentum. 2013;23(66)57-70.

4. Mignone J, Gómez JH. Anas Wayuu, el éxito de una organización indígena de salud colombiana en medio de un sistema en crisis. Voces en el Fénix. 2014;5(41):78-85.

5. Departamento Administrativo Nacional de Estadística. Censo General 2005 [Internet]. Colombia [citado 5 ago 2015]. Disponible en: http://goo.gl/ jsqWiH.

6. Ministerio de Salud y Protección Social. Perfil epidemiológico. En: Estudio técnico de servicios diferenciales para los pueblos indígenas de Colombia [citado 24 oct 2015]. Bogotá: Minsalud; 2014. Disponible en: https://goo.gl/jfvoqe. 
7. Pancho A, Bolaños G, Manios S, Chavaco A, Viluche J, Sisc M. Educación superior indígena en Colombia, una apuesta de futuro y esperanza. Cali: Editorial Universidad de San Buenaventura; 2004.

8. Dirección General de Promoción Social. Diagnóstico y caracterización de la población indígena del departamento de la Guajira. Bogotá: Dirección General de Promoción Social, Ministerio de la Protección Social; 2008.

9. Carruyo M. Análisis de la situación de salud de fronteras Páez-Venezuela, 2005-2006: Informe técnico de epidemiología municipal de salud. Caracas: Ministerio del Poder Popular para la Salud; 2010

10. Organización Mundial de la Salud. Medicina tradicional: definiciones [Internet]. Ginebra: OMS [citado 20 may 2016]. Disponible en: http://goo. gl/qUOhgp.

11. Fandiño Y. Violación del estado colombiano del derecho a la salud de los indígenas wayuu, por el suministro de medicina alopática y desconocimiento de su medicina tradicional. Advocatus. 2010;(15):72-78.

12. Mignone J, Nállim J, Gómez Vargas JH. Indigenous control over health care in the midst of neoliberal reforms in Colombia: An uneasy balance. Studies in Political Economy. 2011;87:93-107.

13. González LA. Análisis comparativo de modelos de atención en salud para población indígena en la región Andina. Revista Facultad Nacional de Salud Pública. 2015;33(1):7-14.

14. Ministerio de Salud y Protección Social. Estudio técnico de servicios diferenciales para los pueblos indígenas de Colombia [Internet]. Bogotá: Minsalud; 2014. [citado 3 oct 2015]. Disponible en: https://goo.gl/jfvoqe.

15. Cooper CR, Denner J, López EM. Cultural brokers: Helping Latino children on pathways toward success. The Future of Children. 1999;9(2): 51-57.

16. Musser-Granski J, Carrillo DF. The use of bilingual, bicultural paraprofessionals in mental health services: issues for hiring, training, and supervision. Community Mental Health Journal. 1997;33(1):51-60.

17. Singh NN, McKay JD, Singh AN. The need for cultural brokers in mental health services. Journal of Child and Family Studies. 1999;8(1):1-10.

18. Gregg J, Saha S. Communicative competence: A framework for understanding language barriers in Health Care. Journal of General Internal Medicine. 2007;22(Suppl 2):368-370.

19. Kaufert JM, Koolage WW. Role conflict among 'culture brokers': The experience of native Canadian medical interpreters. Social Science \& Medicine. 1984;18(3):283-286.

20. Flores G. The impact of medical interpreter services on the quality of health care: A systematic review. Medical Care Research and Review. 2005;62(3):255-299.

21. Mignone J. Latin American community-based mental health initiative: Stakeholder Matrix Tool. Journal of Community Psychology. 2002;30(3): 235-245.

22. Egli EA. Bilingual workers. In: Westermeyer J, Williams CL, Nguyen AN, (eds.). Mental health services for refugees (DHHS Publication No. ADM 91-1824). Washington DC: U.S. Government Printing Office; 1991. p. 90-110.

23. Organización Mundial de la Salud. Ampliación de la protección social en materia de salud: Iniciativa conjunta de la Organización Panamericana de la Salud y la Organización Internacional del Trabajo [Internet]. Washington DC: OMS, OIT; 2002 [citado 10 oct 2015]. Disponible en: http:// goo.gl/yWUSJl.

24. Corbin J, Strauss A. Bases de la investigación cualitativa, técnicas y procedimientos para desarrollar la teoría fundamentada. Medellín: Editorial Universidad de Antioquia; 2002.

25. Rosado J, Moreno M. Farmacopea guajira: el uso de las plantas medicinales xerofíticas por la etnia wayuu. Revista CENIC, Ciencias Biológicas. 2010;41:1-10.

26. García N. Estigmatización y exclusión del cuerpo enfermo y anciano. En: Segovia Y, Nates B, (comp.). Territorios, identidades y violencias. Bogotá: Universidad de los Andes; 2011.

27. Kroeger A. South American Indians between traditional and modern health services in rural Ecuador. Bulletin of the Pan-American Health Organization 1982;16(3):242-254.

28. Rodríguez L. Factores sociales y culturales determinantes en salud: la cultura como fuerza para incidir en cambios en políticas de salud sexual y reproductiva [Internet]. Associação Latino Americana de População [citado 10 oct 2015]. Disponible en: http://goo.gl/4EqQw3.

29. Mignone J, Bartlett J, O'Neil J, Orchard T. Best practices in intercultural health: Five case studies 
in Latin America. Journal of Ethnobiology and Ethnomedicine. 2007;3:31

30. Castro MD. Los determinantes socioculturales en la salud sexual y reproductiva de mujeres indígenas. Umbrales. 2009;(18):205-235.

31. Briseño Maas ML. Construcción de la sexualidad y el género en estudiantes de la Escuela Normal Bilingüe e Intercultural de Oaxaca (ENBIO)
[Internet]. Universidad Nacional Autónoma de México; 2011 [citado 26 nov 2015]. Disponible en: http://goo.gl/owWDDN.

32. Banco de la República, Museo del Oro. Pütchipü'ü: el oficio de la palabra entre los wayuu [Internet]. Bogotá: Banco de la República, Museo del Oro [citado 25 nov 2015]. Disponible en: http://goo.gl/vprDIW .

\section{FORMA DE CITAR}

Patiño-Londoño SY, Mignone J, Castro-Arroyave DM, Gómez Valencia N, Rojas Arbeláez CA. Guías bilingües: una estrategia para disminuir las barreras culturales en el acceso y la atención en salud de las comunidades wayuu de Maicao, Colombia. Salud Colectiva. 2016;12(3):415-428. doi: 10.18294/sc.2016.883.

Recibido: 3 de marzo de 2016 | Versión final: 9 de junio de 2016 | Aprobado: 1 de julio de 2016

Este obra está bajo una licencia de Creative Commons Reconocimiento-NoComercial 4.0 Internacional. Reconocimiento - Permite copiar, distribuir y comunicar públicamente la obra. A cambio, se debe reconocer y citar al autor original. No Comercial - Esta obra no puede ser utilizada con finalidades comerciales, a menos que se obtenga el permiso. 